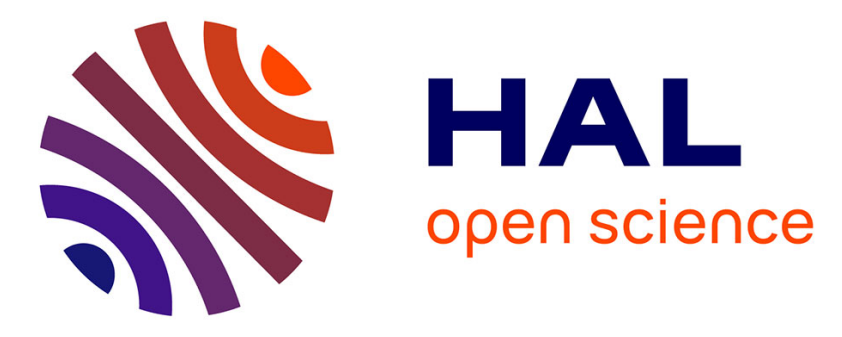

\title{
Identification of scratch mechanisms on a retrieved metallic femoral head
}

Denis Najjar, Maxence Bigerelle, Henri Migaud, Alain Iost

\section{To cite this version:}

Denis Najjar, Maxence Bigerelle, Henri Migaud, Alain Iost. Identification of scratch mechanisms on a retrieved metallic femoral head. Wear, 2005, 258 (1-4), pp.240-250. 10.1016/j.wear.2004.09.035 . hal-03169130

\section{HAL Id: hal-03169130 \\ https://hal.science/hal-03169130}

Submitted on 15 Mar 2021

HAL is a multi-disciplinary open access archive for the deposit and dissemination of scientific research documents, whether they are published or not. The documents may come from teaching and research institutions in France or abroad, or from public or private research centers.
L'archive ouverte pluridisciplinaire HAL, est destinée au dépôt et à la diffusion de documents scientifiques de niveau recherche, publiés ou non, émanant des établissements d'enseignement et de recherche français ou étrangers, des laboratoires publics ou privés. 


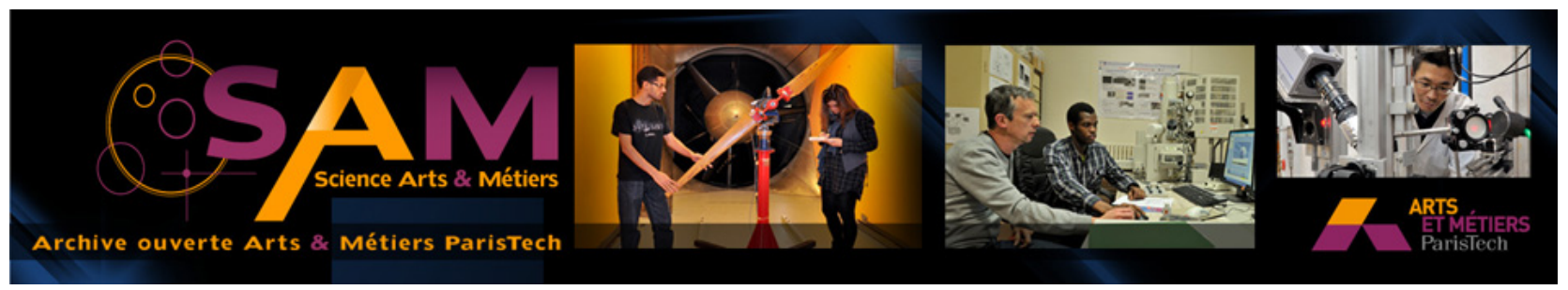

Science Arts \& Métiers (SAM)

is an open access repository that collects the work of Arts et Métiers ParisTech researchers and makes it freely available over the web where possible.

This is an author-deposited version published in: http://sam.ensam.eu Handle ID: .http://hdl.handle.net/null

\section{To cite this version :}

Denis NAJJAR, Maxence BIGERELLE, Henri MIGAUD, Alain IOST - Identification of scratch mechanisms on a retrieved metallic femoral head - Wear - Vol. 258, n¹-4, p.240-250 - 2005 


\title{
Identification of scratch mechanisms on a retrieved metallic femoral head
}

\author{
D. Najjar ${ }^{\mathrm{a}, *}$, M. Bigerelle ${ }^{\mathrm{a}, \mathrm{b}}, \mathrm{H}$. Migaud $^{\mathrm{c}}$, A. Iost $^{\mathrm{a}}$ \\ ${ }^{a}$ Laboratoire de Métallurgie Physique et Génie des Matériaux, UMR CNRS 8517, ENSAM, Equipe Surfaces et Interfaces, \\ 8 Boulevard Louis XIV, 59046 Lille, Cedex, France \\ ${ }^{\mathrm{b}}$ Laboratoire Roberval, FRE 2833, UTC/CNRS, Centre de Recherches de Royallieu, BP20529, 60250 Compiègne, France \\ ${ }^{\mathrm{c}}$ CHRU Lille, Service d'Orthopédie C, Hôpital Roger Salengro, 59037 Lille, Cedex, France
}

\begin{abstract}
The degradation of the femoral head roughness caused by scratching is known to increase the wear rate of the polyethylene counterface of the Charnley total hip prostheses and therefore to have an impact on osteolysis. The knowledge of the scratching mechanism is hence of paramount importance to improve the long-term clinical performance of these prostheses.

The aim of this study is to identify the mechanism of scratch generation of a retrieved titanium (TA6V) femoral head through an accurate characterisation of its surface topography. The surface topography features have been assessed both qualitatively and quantitatively by respectively using an optical microscope and a three dimensional contacting profilometer.

Based on the scanning of about 80 areas spread over the entire surface of the retrieved femoral head, hypotheses on the mechanism of scratch generation have been formulated after analysing the observed topographical patterns, the arithmetic mean roughness and the amplitude density function (ADF). The formulated hypotheses have finally been verified comparing the ADF and the bearing area curves of profiles related to the most severely scratched areas with those of simulated worn profiles. This study highlights the major influence of foreign third bodies on the scratching of metallic femoral heads of the Charnley type prostheses.
\end{abstract}

Keywords: Wear; Femoral head; Scratching mechanism; Roughness parameters; Simulation

\section{Introduction}

The implantation of hip joint prostheses is currently performed world-wide at a rate of approximately 1000 per day and it is estimated that $80 \%$ of these total hip replacement (THR) joints are successful over between 15 and 20 years $[1,2]$. In fact, only a minority of patients who experience high-wear rates remains a source of problems while this type of surgery is in most cases quite successful. Based on this clinical reality, Brown et al. [3] rightly claimed that reducing the cohort's average wear rate is no assurance of correspond-

\footnotetext{
* Corresponding author. Tel.: +33320622 765; fax: +33 320622957 . E-mail address: denis.najjar@lille.ensam.fr (D. Najjar).
}

ing reductions for the problematic minority of patients who suffer high-wear. Conversely, they concluded that it may be fruitful to find solutions to reduce the high-wear situations, even if corresponding improvements in average wear rate happen to be inappreciable.

For the last 30 years, the most implanted prostheses have consisted of a hard metallic or ceramic femoral head articulating against an ultra high molecular weight polyethylene (UHMWPE) acetabular cup. The major concern relating to the long-term performance of these Charnley prostheses is the adverse cellular reactions caused by the generation of UHMWPE wear debris [2,4]. It has been shown that there is a wide distribution in the size of the UHMWPE wear debris in tissues surrounding the Charnley hip prostheses and the mode 
of the frequency distribution is smaller than $1 \mu \mathrm{m}[2,4]$. Furthermore, these micrometer-sized wear debris were found to be the most biologically active $[2,4,5]$ so the reduction of their production rate remains a key issue in order to avoid or to limit the deleterious effects related to their accumulation in the surrounding peri-prosthetic tissues.

While the influence of variations in component design, in surgical technique and/or in patient activity level can not be ignored, the scratching of the metallic femoral head is currently thought to be a major factor affecting the wear rate of the UHMWPE counterface. However, discrepancies have been observed between the results of different laboratory wear tests and clinical observations [3,5-9]. Such discrepancies have mainly been attributed to the fact that localised head scratching may occur in clinical situations knowing that the sites and the size of such damage are seemingly randomly distributed [3,5,7,10]. Brown et al. [3] have recently shown by means of computational results that typical variability in sites and severity of head roughening may account for a significant degree of variability in the wear rate of the UHMWPE counterface.

The scratching of metallic femoral heads of the Charnley type prostheses is increasingly suspected to be caused by hard third bodies such as particles of bone, cement and/or metal, which have often been found embedded in acetabular cups and in the peri-prosthetic tissues $[2,3,5,7,11,12]$. Hence there is an utility in exploring which sites of head scratching tend to be the most deleterious in terms of increasing UHMWPE wear, with a view towards devising steps to impede third body access to these most deleterious head sites [3]. However, due to the stochastic aspects related to the generation, the migration and the characteristics of these hard third bodies, the locations of these deleterious head sites are difficult to predict as well as the point in service life at which roughening occurred [3].

Accurate knowledge of the mechanism behind femoral head scratching is therefore not fully understood in clinical situations, especially in the case of contemporary constructs with modular interfaces and/or porous metal coatings likely to liberate aggressive particulate metal debris. However, this is of prime importance both for an accurate simulation of damaged counterface conditions in laboratory wear tests reproducing relevant clinical topography and for improving the long-term performance of the Charnley total hip prostheses.

The aim of this study is to identify the mechanism behind the scratch generation of a retrieved titanium (TA6V) femoral head through an accurate characterisation of its surface topography. Since the foreign third bodies are increasingly suspected of being the dominant cause of the scratch generation, this titanium femoral head was especially selected because an embedded metallic millimeter-sized fiber was detected in the UHMWPE counterface after revision surgery. This study also aims to contribute to the determination of roughness parameters that should be used in assessing the surface topography of femoral heads in relation to the wear of the UHMWPE cup.

\section{Experimental procedure}

\subsection{Clinical case report}

The articulating components under investigation were retrieved after 4 years and 5 months consequently to the detection of an osteolysis phenomenon (without loosening) on the survey radiographs of the patient. During the period of implantation, the UHMWPE acetabular liner was inserted in a Harris-Galante Mark I metallic cup and the metallic femoral head was mounted on a cemented femoral stem.

After revision surgery, a visual inspection clearly revealed that the retrieved titanium (TA6V) femoral head was severely scratched and that a hard foreign body was embedded in the UHMWPE acetabular liner (Fig. 1a). This foreign body, several millimeters in length, came from the titanium fibermesh which was deposited on the Harris-Galante metallic cup during the fabrication process. It must be pointed out that polyethylene and metallic debris were detected by histological analyses on the peri-prosthetic tissues.

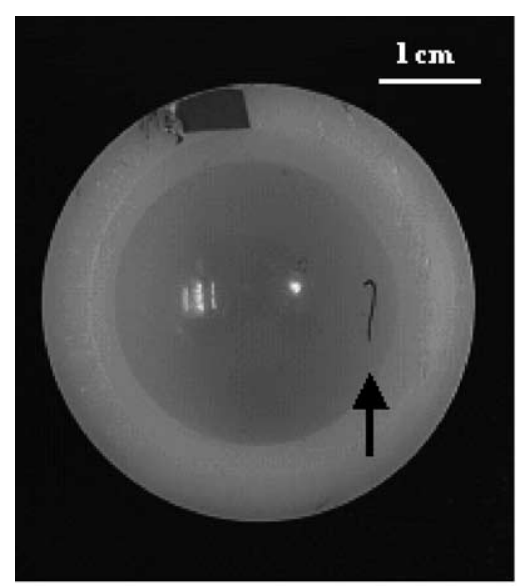

(a)

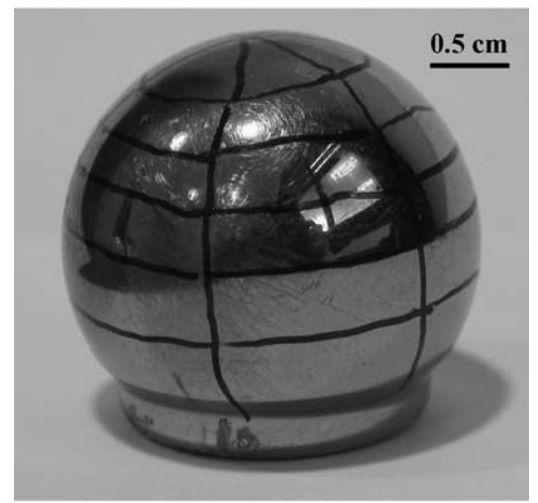

(b)

Fig. 1. Visualisation of (a) the retrieved UHMWPE acetabular liner with an embedded titanium fibermesh piece marked with a black arrow, (b) the retrieved $28 \mathrm{~mm}$ diameter titanium based femoral head and the lines drawn for the reference frame. 
As the degradation mechanism of the UHMWPE acetabular liner has already been described in a previous paper [12], this study mainly focuses on the understanding of the degradation mechanism of the retrieved metallic femoral head through a characterisation of its overall surface topography.

\subsection{Surface topography characterisation}

The qualitative aspect of the damage was characterised combining a visual inspection and observations by optical microscopy. The quantitative measurements were carried out by means of a three dimensional contacting profilometer (KLA Tencor P10) using a $2 \mu \mathrm{m}$ stylus radius and a $2 \mathrm{mg}$ stylus force. Errors related to this experimental technique are presented in Appendix A.

Before these measurements, a reference frame was defined on the metallic femoral head in order to locate each area under examination in relation to the others. Drawn on the metallic femoral head as illustrated in Fig. 1b, this reference frame consisted of 12 circumferential and meridional lines that delimited 36 different zones. In each zone, depending on its size and possible variations in its visual brightness level from one point to another, one or more characteristic areas were scanned by means of the three dimensional contacting profilometer.

The three dimensional topography was characterised for about 80 areas spread over the entire surface of the retrieved femoral head. For each area, 300 traces of $600 \mu \mathrm{m}$ long were recorded with a $2 \mu \mathrm{m}$ space-interval at a scan rate of $500 \mathrm{~Hz}$ giving a data file containing 900,000 data points. Considering these experimental conditions, it can be deduced that the horizontal resolution along a scanning trace and the size of a scanned area were respectively $0.5 \mu \mathrm{m}$ and $600 \times 600 \mu \mathrm{m}^{2}$. In a first time, all the data files were processed by the Mountains ${ }^{\mathrm{TM}}$ software (Mountains, Digital Surf, Besançon, France). By means of an integrated surface filter, this software allowed us to remove the spherical form of the femoral head and, then, to obtain quickly isometric plots of the topography of all the scanned areas. The same data files were also processed by a specific algorithm developed by the authors. Using the SAS ${ }^{\mathrm{TM}}$ (Statistical Analysis System, SAS Institute Inc., Cary, USA) software language, this specific algorithm was written in order to estimate several roughness parameters of interest retained in the present study.

\subsection{Roughness parameters}

In the specific algorithm developed by the authors, the form was removed by means of a fitting method before estimating any roughness parameter of interest. The best-fitting curve selected for this pre-processing operation was a second order polynomial curve whose coefficients are estimated by the least squares method. Fig. 2a presents an example
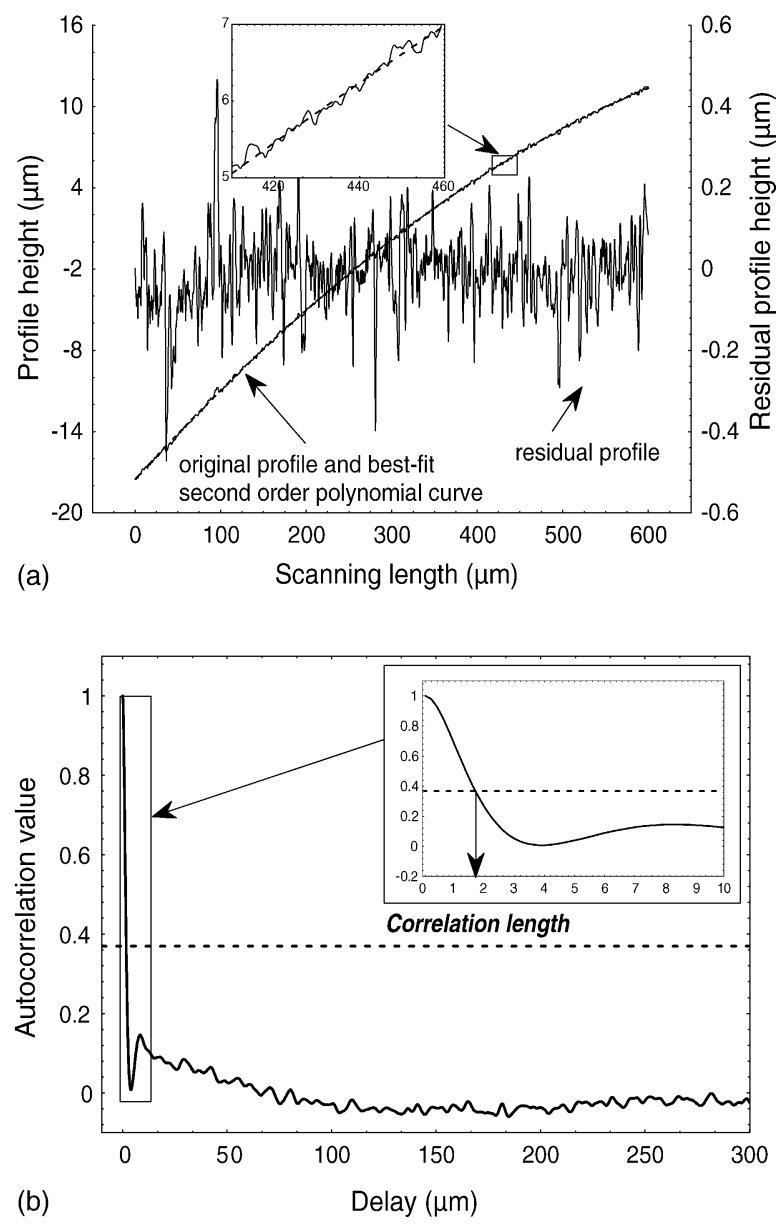

Fig. 2. Example of results showing: (a) a residual profile obtained by removing the form of an experimental profile by means of a best-fitting second order polynomial curve, (b) the related autocorrelation function of the residual profile.

of an original profile, its best-fitting second order polynomial curve and the related residual profile. The relevance of selecting a second order polynomial curve for this preprocessing operation of form removal is a posterior justified: qualitatively, by the high goodness-of-fit that can be noticed between this fitting curve and the original profile and, quantitatively, by analysing the autocorrelation function related to the residual profile. Indeed, it can be seen in Fig. $2 b$ that this aucorrelation function rapidly decays and the related correlation length (defined as the length over which the autocorrelation decays to a fraction of its initial value taken as $1 / e$ ) is extremely short compared to the scanning length.

Then, after this pre-processing operation of form removal, our specific program determines the amplitude density function (ADF) and estimates the average of the arithmetic mean roughness $R_{\mathrm{a}}$ of the 300 residual profiles related to the 300 scanning traces of each recorded data file. Conventionally used as an indicator of femoral head damage in laboratory investigations [3-8,10], the arithmetic mean roughness $R_{\mathrm{a}}$ 


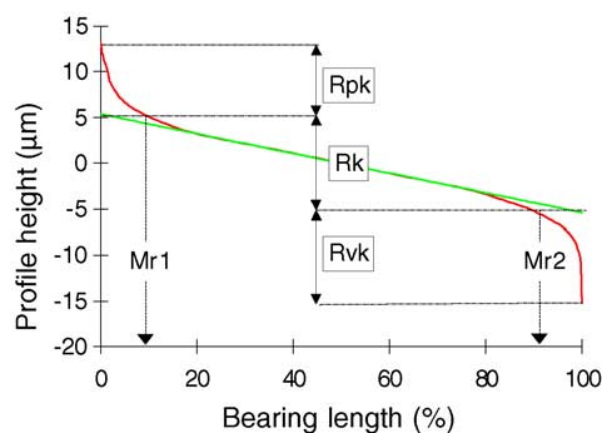

Fig. 3. Schematic representation of a bearing area curve as well as the related roughness parameters $R_{\mathrm{k}}, R \mathrm{pk}, R \mathrm{vk}, \mathrm{Mr}_{1}, \mathrm{Mr}_{2}$.

measures the mean absolute deviation of roughness irregularities from the reference line (the best-fitting second order polynomial curve in this study). Although this roughness parameter is easy to define and to measure, it remains an average parameter which does not contain any information about the spatial and textural variation of the topography, that is to say the variation in height from one point to another. Thus, the $\mathrm{ADF}$ was considered to provide a more detailed and realistic information about the overall topography. Indeed, the ADF represents the distribution histogram of profile heights that can be assessed by plotting on a graph the frequency of profile heights found within a given height $z$ and a height $z+\Delta z$ as function of the given height $z$.

Additionally, the bearing area curves (Abbott-Firestone curves) of some typical scanned areas have also been determined. From a physical point of view, the bearing area curve represents the evolution of the effective contact area as the surface wears out. This curve is obtained by cal- culating the value of the bearing line length parameter $t_{\mathrm{p}}$ at different heights selected on the total amplitude $R_{\mathrm{t}}$ of the profile; 200 bearing levels have been considered in this study.

Fig. 3 shows a schematic example of a bearing area curve having a S-shape appearance as it is the case for many engineering surfaces. The horizontal axis represents the bearing area lengths as a percentage of the total assessment length of the profile and the vertical axis represents the heights of the profile. This figure also presents the roughness parameters $R_{\mathrm{k}}$, $R_{\mathrm{pk}}, R_{\mathrm{vk}}, \mathrm{Mr}_{1}$ and $\mathrm{Mr}_{2}$ that can be inferred from the bearing area curve according to the guidelines mentioned in the DIN 4776 and ISO $13565-2$ standards $[13,14]$. The parameter $R_{\mathrm{k}}$ is called the core depth which measures the height of the core material. The parameter $R_{\mathrm{pk}}$ is called the reduced peak height which denotes the portion of the protuding peaks above the core profile. The parameter $R_{\mathrm{vk}}$ is called the reduced valley depth which denotes the portion of deep valleys extending into the material below the core profile. The parameter $\mathrm{Mr}_{1}$ represents the material ratio at the transition between protuding peaks and core. Finally, the parameter $\mathrm{Mr}_{2}$ represents the material ratio at the transition between core and deep valleys.

\section{Experimental results and discussion}

\subsection{Assessment of the topographical patterns}

Fig. 4 summarises the results obtained combining a visual inspection and observations by optical microscopy. Three kinds of regions can be observed on the retrieved titanium femoral head under consideration:
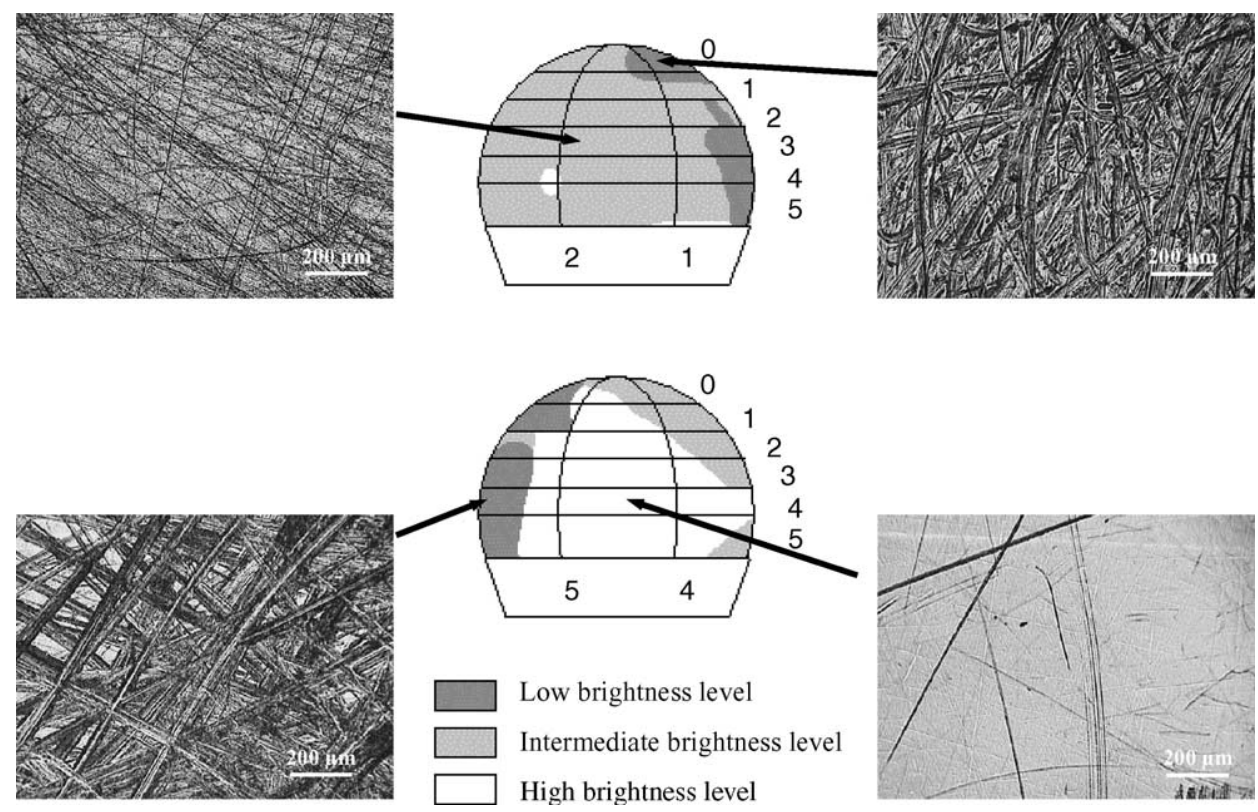

Fig. 4. Summary of the qualitative results obtained combining a visual inspection and observations carried out by means of an optical microscope. 
- regions covering about $30 \%$ of the femoral head surface and visually having a bright finish. A light degree of damage is noticed in these regions which contain only isolated scratches.

- Regions covering between 10 and $20 \%$ of the femoral head surface and visually having a low brightness level; two regions of this type were detected and located along a meridian. A severe degree of damage is noticed in these regions whose topography consists of a high density of large, deep and randomly oriented scratches. These large scratches are thought to be directly related to the third-body abrasive action of the metallic millimeter-sized fiber on the metallic femoral head, consequently producing numerous metallic micrometer-sized wear debris.

- Regions covering more than $50 \%$ of the femoral head surface and visually having an intermediate brightness level. A moderate degree of damage is noticed in these regions whose topography consists of a high density of numerous small and randomly oriented scratches. These numerous small scratches are thought to be mainly a kind of "collateral damage" due to the third-body abrasive action of the small metallic micrometer-sized wear debris generated by the friction of the metallic fiber against the femoral head.

The topographical patterns related to these three kinds of regions can also be observed on the isometric plots of typical areas recorded by means of the three dimensional contacting profilometer. Examples of such typical isometric plots are presented in Fig. 5 for areas that originate from regions having respectively a high, an intermediate and a low brightness levels. As far as the areas originating from regions having a low brightness level are concerned, the surface topogra-

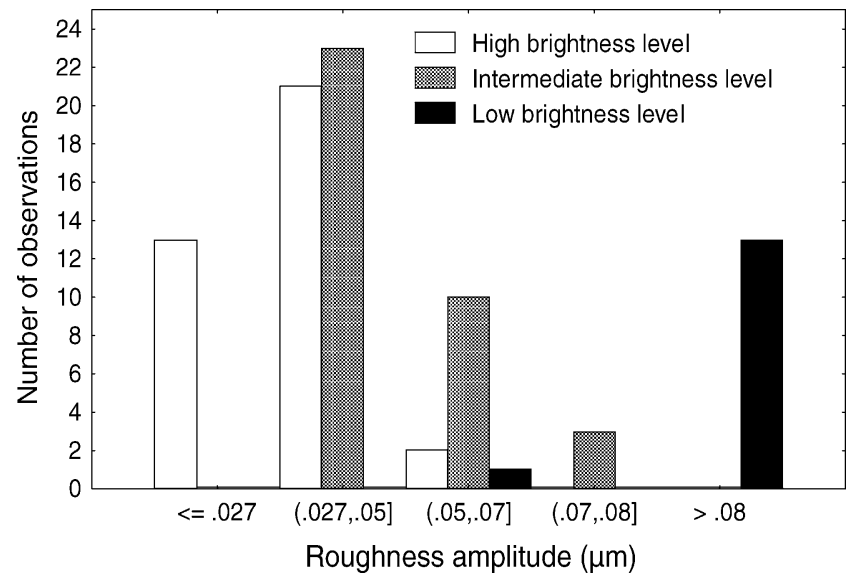

Fig. 6. Histogram of the arithmetic mean roughness values recorded from the areas scanned over the entire surface of the retrieved femoral head.

phy always consists of large scratches superimposed onto a background of small scratches. Furthermore, pile-up metallic material can be detected to the sides of the large scratches in some areas (Fig. 5c) while not in others (Fig. 5d).

\subsection{Analysis of evolution of the arithmetic mean roughness over the entire surface}

The values of the arithmetic mean roughness of the 86 areas scanned by means of the contacting profilometer are presented in Fig. 6. Fifty seven of these reported values are lower than $0.05 \mu \mathrm{m}$ which is the upper limit tolerance mentioned in the standard ISO 7206-2 for the roughness of a metallic femoral head [15]. However, about $50 \%$ of
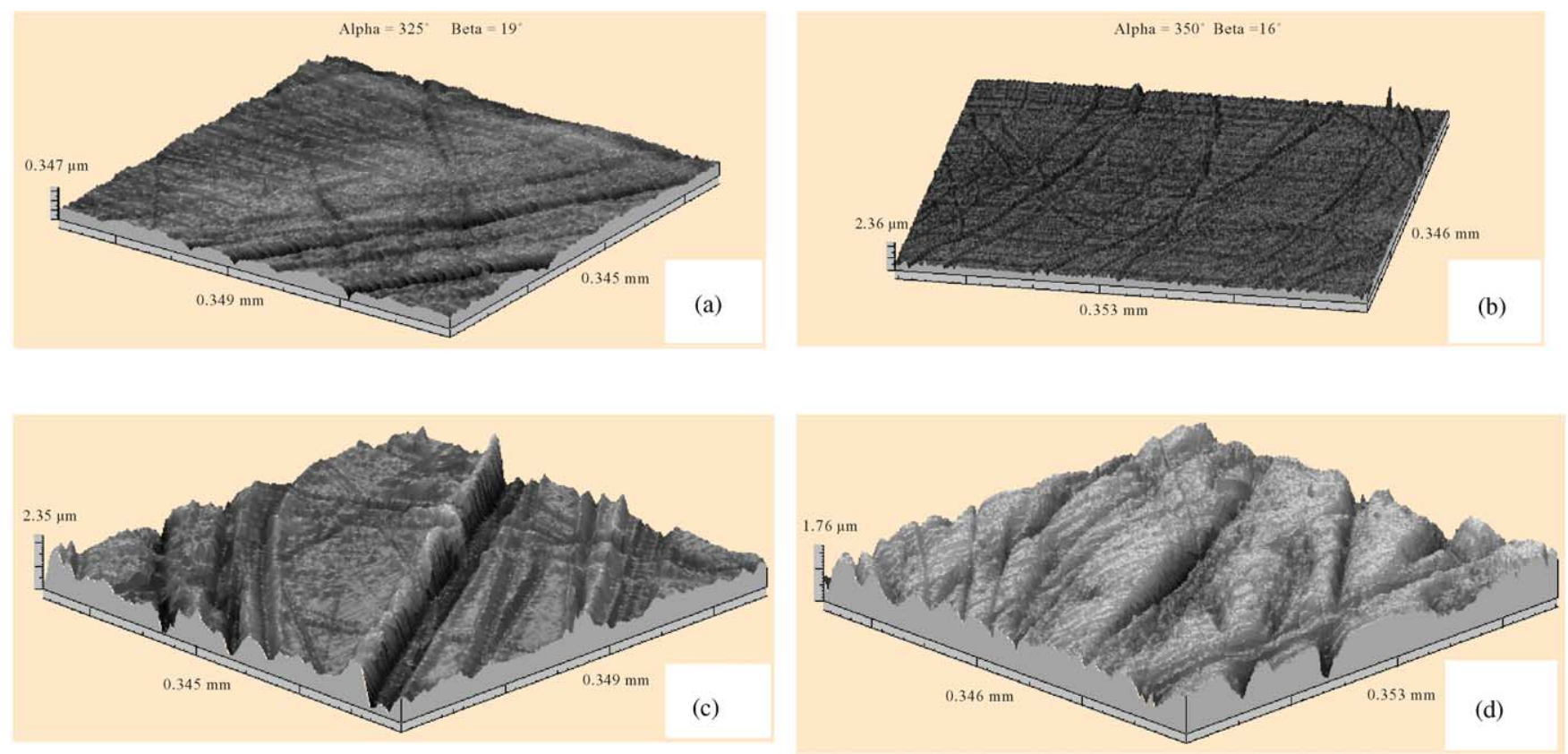

Fig. 5. Isometric plots of typical areas having: (a) a high brightness level, (b) an intermediate brightness level, (c) a low brightness level and large scratches with pile-up material to the sides, (d) a low brightness level and large scratches without any pile-up material. These areas are respectively denoted $5 \mathrm{~A}, 5 \mathrm{~B}, 5 \mathrm{C}$ and $5 \mathrm{D}$. 
these values are related to areas having an intermediate brightness level; that is to say areas which are moderately damaged.

Most importantly, Fig. 6 clearly shows a breakdown value of $0.08 \mu \mathrm{m}$ above which all the reported values of the arithmetic mean roughness parameter are related to areas having a low brightness level. This means that the arithmetic mean roughness parameter can be used to discriminate areas which are severely damaged against the others. However, most of the reported values of this parameter (exactly seventy three values) fall below $0.08 \mu \mathrm{m}$ and, except in one case, all of them are related to areas having either a bright finish or an intermediate brightness level. Unfortunately, no breakdown value can be clearly identified for separating these areas which are either lightly or moderately damaged. In this range of values, the gentle evolution noticed on the figure indicates that the arithmetic mean roughness may not be sufficiently relevant for a significant statistical discrimination of these two kinds of areas.

In other words, this average roughness parameter only allowed a rough discrimination of areas having quite significant brightness levels and roughness amplitudes. However, as far as the severely damaged areas are concerned, this average roughness parameter failed to discriminate surface topographies whose patterns are still quite different.

\subsection{Analysis of the ADF and the bearing area curves of typical areas}

While the ADF was also assessed for each scanned area, Fig. 7 only provides examples of the typical distribution histograms of profile heights, which are representative of the overall areas. In fact, the four selected distributions are precisely those corresponding to isometric plots presented in Fig. 5, that is to say distributions characterising the four typical kinds of surface topography encountered in this study. The bearing area curves related to these typical areas are also given in Fig. 8 and the roughness parameters deduced from these curves are reported in Table 1.

Except for the distribution related to the severely damaged areas containing large scratches without pile-up material to the sides, all the distributions are symmetric compared to the reference surface and approach the Gaussian distribution (Fig. 7). Therefore, for these three kinds of area, there are as many peaks as valleys about the reference surface and these features are consistent with a random damage process. More-
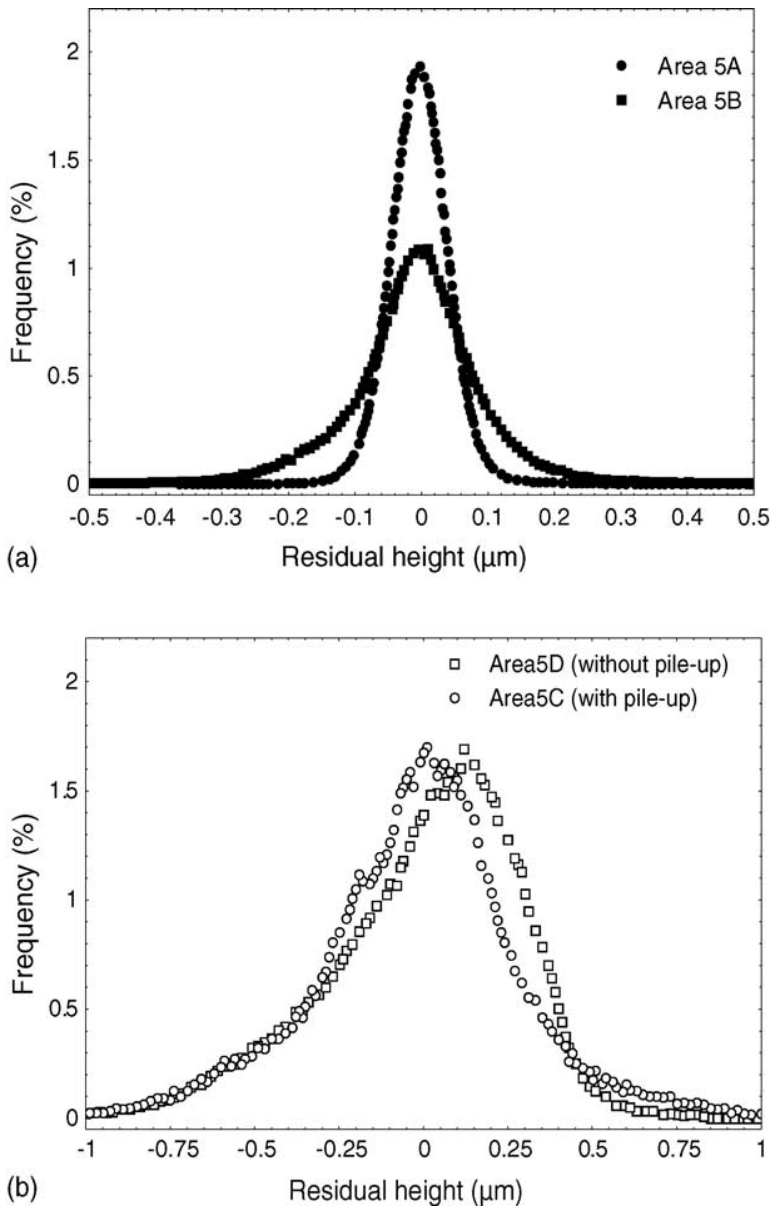

Fig. 7. Amplitude Density Functions of the four typical areas: (a) 5A and $5 \mathrm{~B}$, (b) $5 \mathrm{C}$ and $5 \mathrm{D}$

over, it can be noticed that the standard deviation of the ADF increases, as the severity of the damage is more important. This simply means that, as expected, the height of the peaks and the depth of the valleys further increase with the degree of damage. This qualitative result is quantitatively confirmed by the fact that the amplitude parameters $R_{\mathrm{pk}}$ and $R_{\mathrm{vk}}$ deduced from the related bearing area curves (Fig. 8) increase as the amplitude mean roughness $R_{\mathrm{a}}$ increases. Besides, it is worth noting that the values of the roughness parameters $\mathrm{Mr}_{1}$ and $\mathrm{Mr}_{2}$ (which respectively characterise the lower and the upper limits of the core roughness) are statistically the same whatever the amplitude roughness of the considered area. These results may suggest that the topographies of these different areas have the same texture to within the roughness amplitude

Table 1

Values of the roughness parameters calculated from the original and the modified bearing area curves of the four typical areas 5A, 5B, 5C and 5D

\begin{tabular}{llllllllrr}
\hline Area & $R_{\mathrm{a}}$ & $R_{\mathrm{q}}$ & $R_{\mathrm{pk}}$ & $R_{\mathrm{k}}$ & $\mathrm{R}_{\mathrm{vk}}$ & $\mathrm{Mr}_{1}$ & $\mathrm{Mr}_{2}$ & $R_{\mathrm{pk}}^{*}(\%)$ & $R_{\mathrm{k}}^{*}(\%)$ \\
\hline 5A & 0.03 & 0.05 & 0.56 & 0.11 & 0.31 & 10.21 & 90.09 & 58 & 11 \\
5B & 0.07 & 0.10 & 1.78 & 0.21 & 0.94 & 11.12 & 87.46 & 61 & 7 \\
5C & 0.24 & 0.34 & 2.37 & 0.65 & 0.92 & 11.57 & 86.36 & 61 & 16 \\
5D & 0.23 & 0.29 & 0.74 & 0.66 & 1.27 & 6.27 & 83.96 & 27 & 32 \\
\hline
\end{tabular}




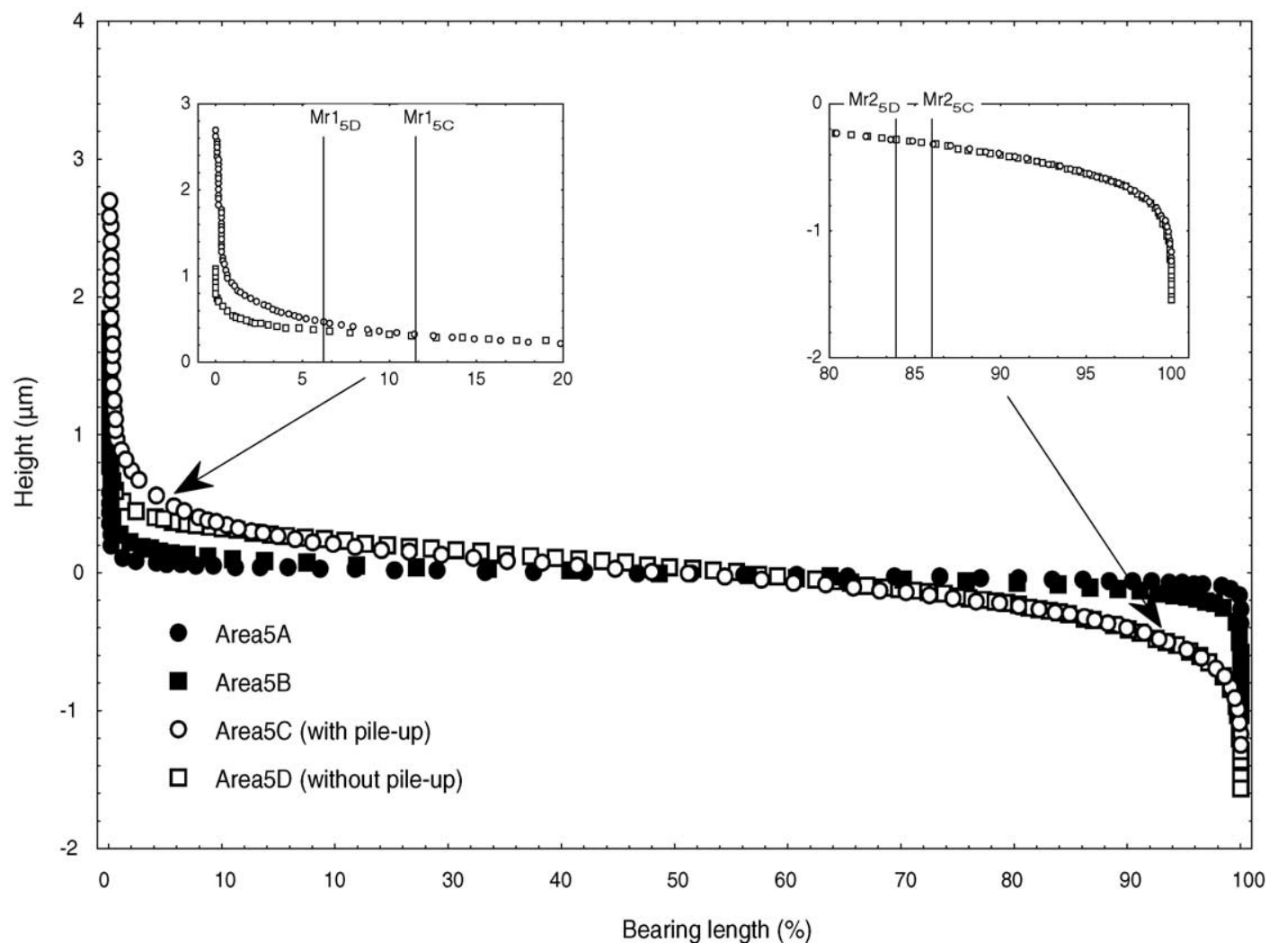

Fig. 8. Bearing area curves of the four typical areas 5A, 5B, 5C and 5D.

scale, which means the surface topography of these damaged areas seems to follow a scaling law.

In order to verify the previous assumption, a modified bearing area curve was constructed for each area under consideration by dividing the original values of the heights reported on the vertical axis by the root-mean square roughness $R_{\mathrm{q}}$ calculated from the related ADF. Fig. 9 shows that all the modified bearing area curves are quite similar as well as the related dimensionless roughness parameters $R_{\mathrm{pk}}^{*}, R_{\mathrm{k}}^{*}$ and $R_{\mathrm{vk}}^{*}$

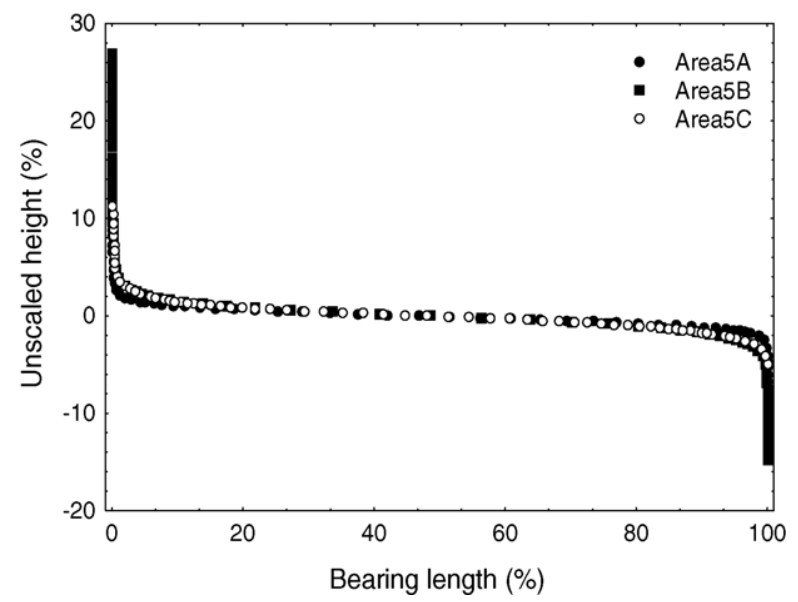

Fig. 9. Modified bearing area curves of the typical areas 5A, 5B and 5C. that can be extracted from these curves (Table 1). This result thus seems to confirm that the surface topography of these damaged areas follows a scaling law.

As far as the severely damaged areas are concerned, it is very interesting to notice that, independently of the existence or the absence of pile-up material to the sides of the large scratches, the values of the arithmetic mean roughness $R_{\mathrm{a}}$ are the same. This means that this roughness parameter is not sufficient to discriminate the surface topographies of these areas whose patterns are however quite different. Conversely, as shown in Figs. 7 and 8, the shapes of the ADF as well as the bearing area curves are significantly different for the two areas under study. Indeed, the ADF related to the area containing large scratches without pile-up material is asymmetric and negatively skewed (Fig. 7) while the ADF related to the area containing large scratches with pile-up material to the sides is symmetric. In the same way, it is worth noting that the bearing area curves related to these areas are different for bearing lengths $t_{\mathrm{p}}$ lower than approximately $12 \%$ while they are identical above this value. These qualitative results are logically reflected in the values of the roughness parameters that were deduced from these curves and reported in Table 1. Indeed, it can be noticed that, while the values of $R_{\mathrm{vk}}, R_{\mathrm{k}}$ and $\mathrm{Mr}_{2}$ are quite similar, the values of $R_{\mathrm{pk}}$ and $\mathrm{Mr}_{1}$ related to the area containing large scratches with pile-up material are significantly higher than that of those related to the area containing large scratches without pile-up material. These results 
physically indicate that, while the regimes related to the core roughness and to the valleys lying below the core roughness are identical for the two considered areas, the values of the amplitude of the peaks above the core roughness as well as the values of the density of these peaks related to the area containing large scratches with pile-up material are higher than those related to the area containing large scratches without pile-up material. These results may suggest that the scratching process over these areas is the same but the absence of pile-up material along the edges of large scratches in some areas may be the consequence of a wear process that continuously removed existing head scratches leaving the deep valleys unaffected.

In order to verify this new assumption, an algorithm simulating an elementary abrasive wear process was specifically developed and applied on the experimental data file related to the severely damaged area containing large scratches with pile-up material to the sides. The basic idea of this model is that the higher the height of a peak of the profile, the lower its probability of resistance during a wear cycle. This probability of resistance is assessed assuming an exponential decay of the related probability density function, which is characterised by a single parameter defining the magnitude of the wear process. Finally, the probability to remove a peak at a given height is simply obtained by subtracting its probability of resistance from 1. Based on a Monte-Carlo process, the details of this algorithm which simulates the effect of an abrasive wear process that acts continuously to remove the highest peaks of the surface topography are reported in Appendix B. Provided an optimisation of the parameters of this elementary wear model by means of a $\chi^{2}$ calculation, a satisfying goodness-of-fit can be obtained between the bearing area curves related to the processed data file and to the severely damaged area containing large scratches without pile-up material (Fig. 10). Moreover, while the ADF of original data file is symmetric, the $\mathrm{ADF}$ of the processed data file is negatively skewed like the ADF related to severely damaged area con-

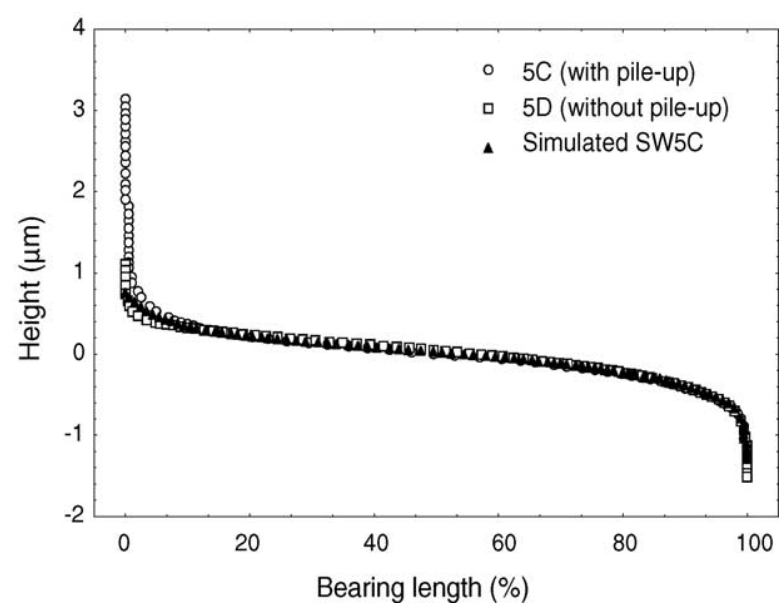

Fig. 10. Bearing area curves of the simulated worn area SW5C compared with those of the original areas $5 \mathrm{C}$ and $5 \mathrm{D}$.

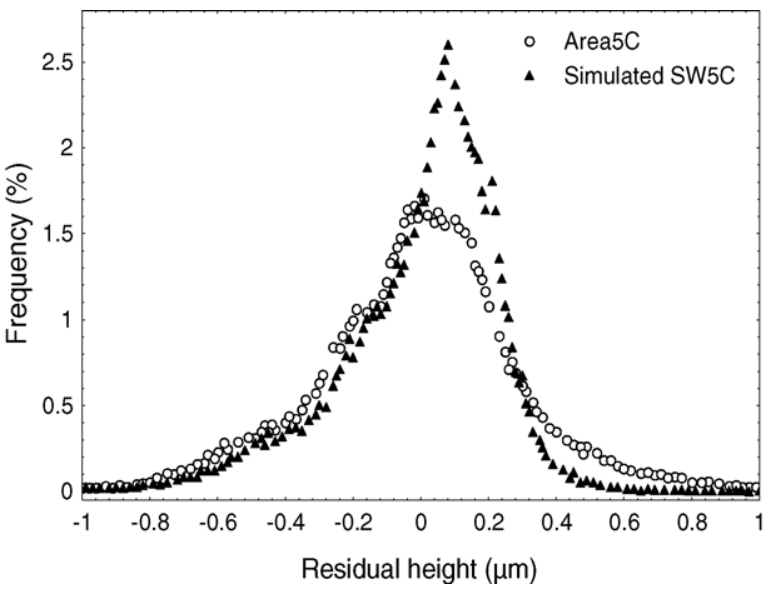

Fig. 11. Amplitude density functions of the simulated worn area SW5C compared with that of the original area $5 \mathrm{C}$. Note the asymmetric shape of this $\mathrm{ADF}$ while that related to the original area $5 \mathrm{C}$ was symmetric before applying the simulated wear process.

taining large scratches without any pile-up material (Fig. 11). Therefore, these results confirm that an abrasive wear process may have acted continuously in some severely damaged areas, consequently removing the highest peaks while leaving the deepest valleys unaffected. In fact, this continuously acting wear process is strongly believed to be caused by multiple passages of the metallic fibermesh piece over the areas under consideration.

\subsection{Scratching mechanism of the femoral head and its consequences}

The overall results of the present investigation show that the metallic millimeter-sized fibermesh piece migrated into the effective joint space where it continued moving and participated in the deleterious abrasive third-body wear process, consequently affecting the roughness of the retrieved femoral head and probably contributing to the increased wear of the polyethylene acetabular liner. These results are entirely consistent with the viewpoint that in vivo scratching of metallic femoral heads of the Charnley type prostheses is increasingly suspected to be caused by hard abrasive foreign particles which act as third bodies [2,3,5,7,11].

Since contemporary constructs with modular interfaces and/or porous metal coatings are prone to liberate aggressive foreign third bodies, their access to the most deleterious sites of femoral heads have to be impeded. To the knowledge of the authors, if some retrieval studies revealed that, in clinical situations, the sites and the size of worn areas on metallic femoral heads are seemingly randomly distributed $[3,5,7,10]$, any of them precisely discusses the effect of embedded foreign third bodies on the characteristics of surface roughening. The present study hence constitutes an original attempt to characterise accurately the severity of head roughening over the entire surface of a retrieved titanium femoral head. 
The heights of the scratches detected on the investigated femoral head are similar to those reported in other retrieval clinical cases in which clinically relevant scratches on metallic femoral heads have been found to range from lower than $0.1 \mu \mathrm{m}$ to higher than $1 \mu \mathrm{m}[2,10,11]$. Moreover, in agreement with the viewpoint of McNie et al. [11], it is believed that the largest scratches observed on the retrieved femoral head have the highest contribution to the increased wear of the UHMWPE counterface although the effect of the smallest scratches cannot be ignored. Furthermore, it was shown that such large scratches accompanied by pile-up material are primarily responsible for the increased wear rates of the UHMWPE counterface, a major parameter being the scratch lip aspect ratio $[2,7,11]$.

As far as foreign third bodies are concerned in the overall degradation process of components, it would be finally interesting to perform further similar investigations on other retrieved femoral heads to improve our understanding on the characteristics (location, size and topography) of the most deleterious sites.

\section{Conclusions}

A comprehensive identification of scratch topography of a retrieved TA6V femoral head has been proposed through mapping of the surface topography by means of visual inspection, optical microscopy and three dimensional profilometry. This study particularly emphasises the deleterious role of a large-sized metallic foreign body on the damage of the articulating components consequently to its ingress into the effective joint space and its participation to a third body abrasive mechanism. The accurate mapping of damaged areas allows to specify the potent most deleterious head sites in terms of increasing the wear of the UHMWPE counterface. This constitutes a first step to finding solutions to impede the third body access to these most deleterious sites. Similar experimental works on other retrieved femoral heads are however needed to identify general trends on the location, the size and the topography of these potent deleterious sites.

The results of this study have unambiguously revealed some limitations of the arithmetic mean roughness as a relevant indicator of head damage. Indeed, as far as the severely damaged areas of this study are concerned, this average roughness parameter fails to discriminate surface topographies whose patterns are still quite different. Contrary to this average roughness parameter, the ADF and the roughness parameters extracted from the bearing area curve have shown to provide respectively more qualitative and quantitative information about the spatial and textural variation of the surface topography. However, only some roughness parameters that were thought to be relevant were selected for this investigation and, since a surface topography can be characterised by numerous roughness parameters, further work is needed to determine objectively which roughness parameter(s) should be used for describing the effect of texture on the wear of the UHMWPE counterface.

\section{Appendix A}

During scanning of a profile by means of a mechanical profilometer stylus, three types of error are induced. These errors, which are not necessarily independent each other, result from the values of: the stylus load, the scanning speed and the stylus tip radius.

As function of the stylus load, damaging plastic deformation of the profile peaks may occur affecting the measurement of the real profile. In this investigation, no induced damage has been noticed by scanning electron microscopy (SEM) observations of a bright area (i.e. a non scratched area) of the femoral head previously scanned by using a $2 \mathrm{mg}$ stylus load.

As far as the scanning speed is concerned, it should be outlined that the higher its value the higher the probability for the stylus to separate from the surface due to inertia effect. Prior to our measurements, it has been verified that the scanning speed selected had any effect on the recorded profile related to a calibration sample consisting of a step of $1 \mu \mathrm{m}$ (i.e. on a profile having a very unfavourable shape compared to the recorded profiles on the studied femoral head).

Without the knowledge of the real profile, the most difficult error to assess is related to the smoothing effect of this profile due to the stylus tip radius. A computer algorithm has been developed to simulate the effect of a 5 or $10 \mu \mathrm{m}$ stylus tip radius on experimental profiles recorded with a $2 \mu \mathrm{m}$ stylus tip radius in areas denoted 5A, 5B, 5C and 5D. Fig. A1 presents the evolution of the bearing area curves of the four studied areas as function of the stylus tip radius. Whatever the studied area, it can be clearly noticed that the curves are similar meaning that the effect of stylus tip radius can be neglected with regard to the roughness amplitude.

In summary, these three types of errors have been minimised over the experimental conditions of this investigation.

\section{Appendix B}

This simplistic model was used to simulate a wear process without any particular physical specifications. Let the total amplitude be $R_{\mathrm{t}}=z_{\max }-z_{\min }$ where $z_{\max }$ and $z_{\min }$ are respectively the maximum and the minimum heights of the profile. Then, we define the probability $R_{\lambda}(x, c)$ for a small piece of material of height $\mathrm{d} h$ located at position $x$ to resist to wear during a cycle denoted $c$ as follows:

$$
R_{\lambda}(x, c)=k \int_{0}^{R_{\mathrm{t}}(c)-z^{\prime}(x, c)} \mathrm{e}^{-\lambda h} \mathrm{~d} h
$$

with $k$ and $\lambda$ being real numbers and $z^{\prime}$ ranging from 0 to $R_{t}(c)$ for each cycle $c$. The higher the value of $\lambda$, the higher 

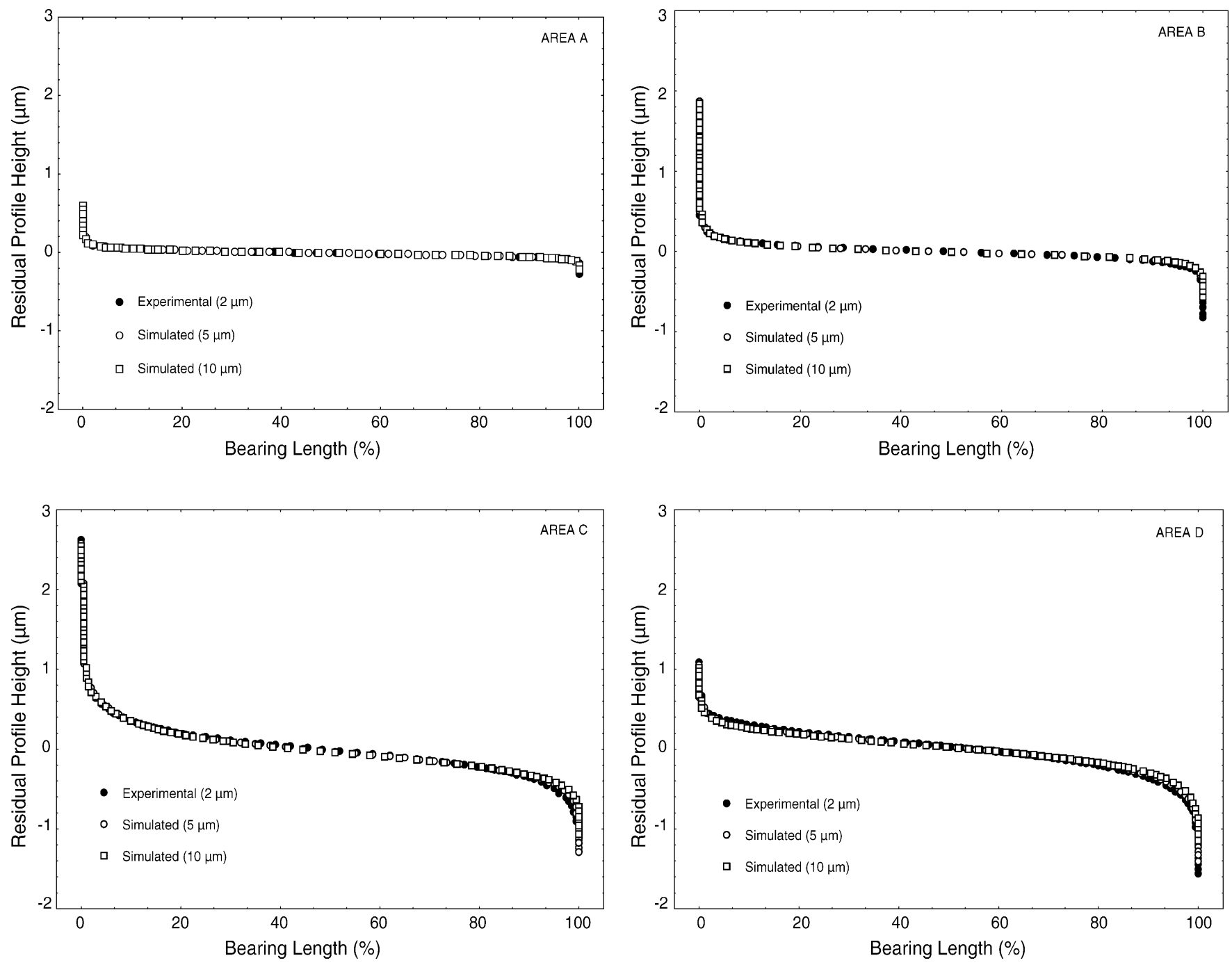

Fig. A1. Effect of a simulated 5 or $10 \mu \mathrm{m}$ stylus tip radius on the bearing area curves of the four typical areas scanned by means of a three dimensional profilometer having a $2 \mu \mathrm{m}$ stylus tip radius.

the magnitude of the wear phenomenon. This resistance wear integral is then normalised to obtain a probability density function from which the following expression of $k$ can be deduced:

$k=\left[\frac{1-\mathrm{e}^{-\lambda R_{\mathrm{t}}}}{\lambda}\right]^{-1}$

Finally, the probability for an elementary volume of material located at position $x$ and at height $z^{\prime}$ to be eroded during a wear cycle $c$ can be expressed as:

$W_{\lambda}(x, c)=1-\left(\frac{1-\mathrm{e}^{-\lambda\left(R_{\mathrm{t}}(c)-z^{\prime}(x, c)\right)}}{1-\mathrm{e}^{-\lambda R_{\mathrm{t}}(c)}}\right)$

To simulate the wear process, the following algorithm has been applied: At each wear cycle $c$, the value of $R \mathrm{t}(\mathrm{c})$ is computed as well as the value of $W_{\lambda}(x, c)$ estimated for each discretised co-ordinate $x$. Then, a random number $r \in[0 \ldots 1]$ is generated and compared with the values $W_{\lambda}(x, c)$ previously calculated. If $r<W_{\lambda}(x, c)$, a piece of material of height $\Delta h$ is removed from the profile and nothing is done conversely. A new cycle is considered and the different steps described above are repeated.

In this study, the value of $\Delta h(=0.08 \mu \mathrm{m})$ has been selected so as to be small enough to simulate a continuous wear process. The parameters of the elementary wear model $\lambda(=5)$ and $c_{\text {end }}(=700$ cycles $)$ were optimised by means of a $\chi^{2}$ calculation in order to obtain a satisfying goodness-of-fit between the bearing area curves under consideration.

\section{References}

[1] J.F. Osborn, The reaction of bone tissue to hydroxyapatite ceramic coatings during the transition from immediate fixation to permanent fixation following total hip replacement, in: D. Muster (Ed.), Biomaterials: Hard Tissue Repair and Replacement, 1992, pp. 171-189.

[2] C.M. McNie, D.C. Barton, E. Ingham, J.L. Tipper, J. Fischer, M.H. Stone, The prediction of polyethylene wear rate and debris mor- 
phology produced by microasperities on femoral heads, J. Mater. Sci. Mater. Med. 11 (2000) 163-174.

[3] T.D. Brown, K.J. Stewart, J.C. Nieman, D.R. Pedersen, J.J. Callaghan, Local head roughening as a factor contributing to variability of total hip wear: a finite element analysis, J. Biomech. Eng. 124 (2002) 691-698.

[4] J.L. Tipper, E. Ingham, J.L. Hailey, A.A. Besong, J. Fisher, B.M. Wroblewski, M.H. Stone, Quantitative analysis of polyethylene wear debris, wear rate and head damage in retrieved Charnley hip prostheses, J. Mater. Sci. Mater. Med. 11 (2000) 117-124.

[5] R.M. Hall, P. Siney, A. Unsworth, B.M. Wroblewski, The effect of surface topography of retrieved femoral heads on the wear of UHMWPE sockets, Med. Eng. Phys. 19 (1997) 711-719.

[6] D. Dowson, M.M. El-Hady Diab, B.J. Gillis, J.R. Atkinson, Influence of counterface topography on the wear of UHMWPE under wet and dry conditions, in polymer wear and its control, in: L.H. Lee (Ed.), American Society Series, vol. 287, 1985, pp. 171-187.

[7] D. Dowson, S. Taheri, N.C. Wallbridge, The role of counterface imperfections in the wear of polyethylene, Wear 119 (1987) 277-293.

[8] A. Wang, A. Essner, V.K. Polineli, C. Stark, J.H. Dumbleton, Lubrication and wear of UHMWPE in total joint replacements, Tribol. Int. 31 (1998) 17-33.

[9] A.P.D. Elfick, S.L. Smith, S.M. Green, A. Unsworth, The quantitative assessment of UHMWPE wear debris produced in hip simulator testing: the influence of head material and roughness, motion and loading, Wear 249 (2001) 517-527.

[10] J.G. Bowsher, J.C. Shelton, A hip simulator study of the influence of patient activity level on the wear of crosslinked polyethylene under smooth and roughened femoral conditions, Wear 259 (2000) $167-179$.

[11] C.M. McNie, D.C. Barton, E. Ingham, J.L. Tipper, J. Fischer, M.H. Stone, Modelling of damage to articulating surfaces by third body particles in total joint replacements, J. Mater. Sci. Mater. Med. 11 (2000) 569-578.

[12] D. Najjar, A. Behnamghader, A. Iost, H. Migaud, Influence of a foreign body on the wear of metallic femoral heads and polyethylene acetabular cups of total hip prostheses, J. Mater. Sci. 35 (2000) 4583-4588.

[13] DIN 4776, Measurement of surface roughness parameters Rk, Rpk, $\mathrm{Rvk}, \mathrm{Mr} 1, \mathrm{Mr} 2$ for the description of the material portion in roughness, Berlin, Germany, Mai 1990.

[14] ISO13565-2, Geometrical product specification (GPS)—surface texture profile method; surface having stratified properties. Part 2: Height characterisation using linear material ratio curve, Geneva, Switzerland, 1996.

[15] ISO7206-2, Implants for surgery-partial and total hip joint prostheses. Part 2: Articulating surfaces made of metallic, ceramic and plastics materials, Geneva, Switzerland, 1996. 